\title{
V. Sonderregelungen zugunsten der Opfer von Menschen- versuchen nach 1989/90: Schlussstrich oder "Falltür" in die Vergangenheit?
}

Während man in Regierungskreisen in den frühen 1980er Jahren bereits mit einem gewissen Stolz auf das Werk der Wiedergutmachung zurückzuschauen begann, entzündete sich auf zivilgesellschaftlicher und auf politischer Ebene Kritik daran, dass einige Gruppen von Opfern nationalsozialistischer Verfolgung keine Entschädigung erhalten hatten. Verschiedene Organisationen, die sich noch viele Jahre nach der Verabschiedung des Bundesentschädigungs-Schlussgesetzes für die Belange der Betroffenen einsetzten, prägten den Begriff der „vergessenen Opfer“. Damit wurde die mangelnde Wahrnehmung des Verfolgtenschicksals in der Öffentlichkeit, vor allem aber in der BEG-Rechtsprechung beklagt. ${ }^{1} \mathrm{Zu}$ den „vergessenen Opfern“ zählten Zwangssterilisierte aufgrund der Erbgesundheitsgesetzgebung von 1933, diejenigen, die als „Asoziale“ oder „Berufsverbrecher" verhaftet und in Konzentrationslager verbracht worden waren, sowie Deserteure bzw. wegen „Wehrkraftzersetzung“Verurteilte. Faktisch benachteiligt waren außerdem „Sinti und Roma“ sowie Homosexuelle. ${ }^{2}$

Auch bei der Wiedergutmachung für Opfer von Humanexperimenten wurde in vielen Fällen mit zweierlei Maß gemessen. Sogenannte Kriminelle wurden von den Fürsorgemaßnahmen zwar nicht grundsätzlich ausgeschlossen, allerdings hatte man sich bereits im Mai 1952 in der ersten Sitzung des Interministeriellen Ausschusses darauf verständigt, dass bei ihnen schärfere Maßstäbe für die Beurteilung angewandt bzw. die Fälle vorerst zurückgestellt werden sollten. ${ }^{3}$ „Die Öffentlichkeit würde es nicht verstehen, wenn asoziale Personen aus Bundesmitteln dotiert werden. ${ }^{4}$ Dieser Satz aus einer Stellungnahme des damaligen Bundesfinanzministers Fritz Schäffer gegenüber dem Bundesjustizministerium von Februar 1953 spricht eine deutliche Sprache und vergegenwärtigt, dass nicht überwundene Vorurteile und Ressentiments den Hintergrund für eine neuerliche Diskriminierung der im Nationalsozialismus verfolgten Minderheiten auf dem Gebiet der Wiedergutmachung bildeten.

In den meisten Fällen handelte es sich bei den „vergessenen Opfern“ allerdings um jene, für die keine Wiedergutmachungsregelungen getroffen wurden. Für die überlebenden Opfer von Menschenversuchen trifft diese Bezeichnung daher nur bedingt zu. Als Gruppe mit einem spezifischen, unverwechselbaren Verfolgungsschicksal wurden die Betroffenen in der Entschädigungsgesetzgebung nicht „vergessen“, sondern vorsätzlich herausgehalten. Die als Provisorium deklarierte Übergangslösung des Kabinettsbeschlusses von 1951 wurde nie von einer anderen Regelung abgelöst, so dass bis heute gilt: Wer Opfer von Menschen-

1 Vgl. dazu das Kapitel „Vom ,Härtefall' zum ,vergessenen Opfer“", in: Goschler, Schuld und Schulden, S. 345-356.

2 Die „Projektgruppe für die vergessenen Opfer des NS-Regimes in Hamburg e.V.“ publizierte 1986 den Band "Verachtet - verfolgt - vernichtet". Bei den von den Nationalsozialisten verfolgten Kommunisten spricht Gottard Jasper nicht von „vergessenen Opfern", sondern von einer "disqualifizierten Widerstandsgruppe“, vgl. Jasper, Die disqualifizierten Opfer.

${ }^{3}$ Interner Aktenvermerk des AA vom 8.5. 1952, über die erste interministerielle Sitzung am 6.5.1952, PA/AA, Abt. II, Bd. 1664.

${ }^{4}$ Schäffer an BMJ, 5. 2.1953, PA/AA, B 81/147. 
versuchen wurde, hat gegenüber der Bundesrepublik keinen Rechtsanspruch auf Entschädigung.

Von offizieller Seite war im Jahr 1963 betont worden, die Bundesregierung habe gezeigt, dass die Durchführung des Kabinettsbeschlusses in dem Geist erfolgt sei, in dem man ihn im Jahr 1951 gefasst habe - immerhin habe die Bundesregierung bereits 14,5 Millionen DM aus Bundesmitteln für die Opfer von Menschenversuchen gezahlt. ${ }^{5}$ Diese positive Bewertung uneingeschränkt zu teilen, fällt rückblickend schwer angesichts einer amtlichen Praxis, die bei vielen Betroffenen in aller Welt Unverständnis, Unmut und herbe Enttäuschung verursachte. Die Tatsache, dass vieles, was im Rahmen des Möglichen gewesen wäre, von staatlicher Seite versäumt, umgangen oder sogar wissentlich unterlassen wurde, gibt vielmehr der Journalistin Dörte von Westernhagen Recht, die in einem Zeit-Dossier über die Wiedergutmachung bilanzierte: „Die Praxis freilich war kein moralisches Ruhmstück. “6

Die strukturellen Gründe für das Versagen vor einem moralischen Anspruch, der im Kabinettsbeschluss von 1951 explizit formuliert worden war, sind vielschichtig, gleichwohl können sie nicht allein als „Entschuldigung“ dafür herhalten, dass in vielen Fällen altes Unrecht mit neuem Unrecht „wiedergutgemacht“ wurde. Es entstanden Gerechtigkeitslücken, die fortwährend nachgebessert werden mussten und zu Opferkonkurrenzen führten. Antragsteller, die aufgrund des Kabinettsbeschlusses Wiedergutmachung beantragt hatten, mussten ein aufwändiges Entschädigungsverfahren durchlaufen, in dem ihr Leid in Prozentzahlen übersetzt und eingestuft wurde. Andere Opfergruppen wie etwa die Mengele-Zwillinge, die Jahrzehnte aufgrund restriktiver Bestimmungen gar keine Entschädigung erhalten hatten, wurden dagegen im Jahr 1986 mittels Pauschalleistungen entschädigt.

Besonders gravierend waren die Unterschiede im Fall der westeuropäischen Opfer von Menschenversuchen. Sie erhielten zwar wesentlich früher Entschädigungsleistungen als ihre osteuropäischen Leidensgenossen. Dafür wurden die Opfer aus Osteuropa aufgrund der weitgehenden Entscheidungsbefugnis des Internationalen Roten Kreuzes mit weit höheren Summen entschädigt. Nach dem Abschluss der Globalabkommen ergaben sich wiederum Spannungsverhältnisse zu den Individualentschädigungen, da die innerstaatlichen Verteilungskriterien in den osteuropäischen Staaten anderen Gesetzmäßigkeiten unterlagen.

Aber letztlich konnte wohl niemand mit einem akzeptablen Ausgleich des ihm angetanen Unrechts rechnen, am wenigsten diejenigen, die gesundheitliche Schäden erlitten hatten. Dies trifft auch für die überlebenden Opfer von Humanexperimenten zu, da die positiven Möglichkeiten, die eine weitsichtigere Auslegung der Fürsorgeregelung von 1951 zugelassen hätten, nicht ausgeschöpft wurden. Anders sah das die Bundesregierung in ihrem Bericht vom 31. Oktober 1986, in dem die Opfer von Menschenversuchen als ausreichend entschädigt bezeichnet wurden. ${ }^{7}$ Und in einer Dokumentation des Bundesfinanz-

\footnotetext{
5 Metz, Fürsorge, S. 297.

${ }^{6}$ Dörte von Westernhagen, „Wiedergutgemacht?“, Die Zeit vom 5. 10. 1984, S. 33. In den 1980er Jahren begann im deutschsprachigen Raum eine aufmerksamere, tiefgründigere Forschung über die Situation der Nachfahren von Nazi-Tätern. In der Bundesrepublik war es insbesondere die Journalistin Dörte von Westernhagen, die sich - selbst betroffen als Tochter eines SS-Offiziers - in Form von Sozialreportagen mit diesem Thema beschäftigte; vgl. auch Westernhagen, Die Kinder der Täter; außerdem Sichrowsky, Schuldig geboren. Auf der anderen Seite begannen in dieser Zeit auch die Untersuchungen über die Nachfahren der Opfer, zum Beispiel Epstein, Kinder des Holocaust.

7 Bericht der Bundesregierung über Wiedergutmachung und Entschädigung für nationalsozialistisches Unrecht sowie über die Lage der Sinti, Roma und verwandter Gruppen, BT-Drucksachen, 10/6287, 31. 10.1986.
} 
ministeriums vom März 1999 wird mit Hinweis auf die 103,8 Milliarden DM, die für die Wiedergutmachung aufgebracht wurden, scheinbar der Beweis erbracht, dass „nahezu alle durch NS-Unrecht verursachten Schäden" erfasst worden seien. ${ }^{8}$

Aufgrund einer Reihe von Initiativen, die sich für die Belange verfolgter Minderheiten einsetzten, wurde die Wiedergutmachungsfrage trotz der beharrlichen Versuche von offizieller Seite, unter das Thema endlich einen Schlussstrich zu ziehen, gerade in den 1980er Jahren erneut zur Diskussion gestellt. ${ }^{9}$ Seit ihrem Einzug in den Bundestag im Jahr 1983 legte die Fraktion der Grünen einen Schwerpunkt ihrer parlamentarischen Initiativen auf Debatten um die Ausweitung des Entschädigungsrechts. In den Jahren 1985 und 1986 brachte sie entsprechende Gesetzesentwürfe „zur Regelung einer angemessenen Versorgung für alle Opfer nationalsozialistischer Verfolgung “ im Bundestag ein. ${ }^{10}$ Eine befriedigende Lösung in Form eines gesetzlich normierten Anspruchs auf Entschädigung, die allen NS-Opfern zu einer späten Anerkennung verholfen hätte, wurde jedoch nicht erreicht. ${ }^{11}$ Die Regierungskoalition aus CDU/CSU und FDP, die das lästige Thema endgültig vom Tisch haben wollte, ${ }^{12}$ konnte sich lediglich zur Einrichtung eines Härtefonds durchringen, aus dem die von der Wiedergutmachungsgesetzgebung ausgeschlossenen Personen - Euthanasie-Geschädigte, Zwangssterilisierte und Homosexuelle - eine einmalige Zahlung von $5000 \mathrm{DM}$ erhalten konnten. Die Entschädigungspraxis bei überlebenden Opfern von Menschenversuchen blieb durch diesen Härtefonds abermals unberührt.

Als die Mengele Twin Organization im Jahr 1985 dem Bundesfinanzministerium 80 Anträge von überlebenden Opfern der Zwillingsexperimente vorlegte, sorgte dies nicht nur in der Öffentlichkeit und im Parlament für Aufsehen; ${ }^{13}$ das Ministerium erhielt vielmehr die Möglichkeit, seine in der bisherigen Entschädigungspraxis äußerst umstrittene Haltung zu überprüfen. Über Jahrzehnte waren die Entschädigungsansprüche der überlebenden Opfer - die meisten waren Kinder, als sie von Josef Mengele im Konzentrationslager Auschwitz zu Menschenversuchen missbraucht wurden ${ }^{14}$ - abgelehnt. Die Begründung lautete, dass diese Versuche in Nürnberg nicht zur Verhandlung gekommen seien und es sich ferner

8 BMF, Dokumentation 3/99, hrsg. vom Bundesministerium der Finanzen, S. 2 und S. 38.

9 Zum Beispiel: Projektgruppe für die vergessenen Opfer des Nazi-Regimes, Hamburg; Roma und Sinti Union, Hamburg; Bund der „Euthanasie“-Geschädigten und Zwangssterilisierten, Detmold; Tagung der Evangelischen Akademie Bad Boll vom 25. bis 27. 11. 1983 zum Thema „Die Bundesrepublik und die Opfer des Nationalsozialismus“; und die von Klaus Dörner veranstaltete Gütersloher Konferenz, dokumentiert in: Dörner (Hrsg.), Gestern minderwertig; sowie das „Projekt zur Durchsetzung von Wiedergutmachungsansprüchen für Opfer der NS-Medizin“ der Karl-Bonhoeffer-Nervenklinik, Berlin 1989-1991.

${ }^{10}$ Antrag vom 17.10.1995, BT-Drucksachen, 10/4040, und Antrag vom 27.6.1986, BT- Drucksachen, 10/5796; so zeigt Hockerts das ruckartig ansteigende parlamentarische Interesse anhand der zunehmenden Einträge unter dem Stichwort „Wiedergutmachung nationalsozialistischen Unrechts“ im Sachregister zu den Verhandlungen des Deutschen Bundestags und Bundesrats auf, vgl. Hockerts, Wiedergutmachung in Deutschland, S. 202, Anm. 149.

$11 \mathrm{Zu}$ den kontroversen Debatten sowie zur Hinhaltetaktik des Bundesfinanzministeriums in diesen Fragen vgl. Goschler, Schuld und Schulden, S. 350-355.

12 "Gemeinsam eine Erlösung vorbereitet", die lageszeitung vom 5. 12. 1987, S. 5.

13 Bericht der Bundesregierung über Wiedergutmachung und Entschädigung für nationalsozialistisches Unrecht sowie die Lage der Sinti, Roma und verwandter Gruppen, BT-Drucksachen, 10/6287, 31. 10.1986.

14 Kubica, Dr. Mengele. 
nur um Blutentnahmen und vergleichende Messungen gehandelt habe. ${ }^{15}$ In einer offiziellen Stellungnahme der deutschen Behörden aus dem Jahr 1969, die angesichts mehrerer osteuropäischer Fälle abgegeben wurde, ist zu lesen, dass „die Frage, ob man für Zwillingsuntersuchungen ein kleines Schmerzensgeld [...] vorsehen soll, zum jetzigen Zeitpunkt nicht erörtert zu werden braucht". 16

Das Finanzministerium nahm die Gelegenheit, ein Stück „versäumter" Wiedergutmachung nachzuholen, auch im Jahr 1985 nicht wahr. Stattdessen hielt es an der fragwürdigen Auffassung fest, dass „Zwillingsexperimente dieser Art“ ebenso wie die „Reihenuntersuchungen " bei Zwergen ${ }^{17}$ keine Versuche im Sinne des Kabinettsbeschlusses von 1951 und damit auch keine gesundheitsschädigenden Ereignisse darstellten. Ein Jahr später kam erneut Bewegung in die Sache. Anlässlich einer Reise nach Israel erhielt Familienministerin Rita Süssmuth die Gelegenheit, mit „Mengele-Zwillingen“ zu sprechen. Bei einem anschlieBenden Treffen mit dem Vorsitzenden der jüdischen Gemeinde in Berlin, Heinz Galinski, zeigte sich Süssmuth von dem seelischen Leid der Überlebenden außerordentlich betroffen und erklärte, dass man die Entschädigungsmöglichkeiten für Zwillingsexperimente untersuchen werde. ${ }^{18}$ Ein halbes Jahr später, im Juni 1986, meldete der Anwalt der Opfer, dass Bonn sich bereit erklärt habe, den Überlebenden der Zwillingsexperimente zwischen 20000 und 25000 DM als ,moralische Wiedergutmachung“ zu zahlen. ${ }^{19}$

Im Jahr 2001 stellte sich endlich auch die Max-Planck-Gesellschaft ihrem schweren Erbe: Als Präsident der Nachfolgeorganisation der Kaiser-Wilhelm-Gesellschaft, die unter anderem den Forschungseifer des Lagerarztes Mengele großzügig unterstützt hatte, entschuldigte sich Professor Hubert Markl bei den Opfern für die menschenverachtende Forschung an Zwillingen. ${ }^{20}$ Für die Überlebenden war dies eine bedeutungsvolle Geste und 56 Jahre nach der Befreiung ein offizielles Schuldanerkenntnis.

15 Weisung des BMF an die zuständige deutsche Vertrauensärztin in Israel vom 5.2.1954, BA, B 126/12553; dieselbe Auffassung wurde 1968 noch einmal im Zusammenhang mit der Wiedergutmachung für überlebende Opfer von Menschenversuchen aus osteuropäischen Staaten bestätigt, vgl. Aide-Mémoire des AA vom 21.10.1968, PA/AA, B 81/631.

${ }^{16} \mathrm{AA}$ an die Deutsche Vertretung bei den Internationalen Organisationen in Genf, 28.3.1969, PA/ AA, B 86/1233.

17 Eine Münchner Anwaltskanzlei hatte sich seit 1960 für die Entschädigungsforderungen von zwölf Antragstellern eingesetzt, die als Zwergwüchsige im KZ Auschwitz von dem Lagerarzt Josef Mengele zu Experimenten herangezogen worden waren. Trotz der wiederholten Aufforderungen, zu diesen Fällen Stellung zu nehmen, zögerte das Bundesfinanzministerium die abschlägige Entscheidung bis 1965 hinaus. Die Begründung lautete, dass „[das] Ziehen gesunder Zähne, [die] Einnahme von Medikamenten, [das] Haarausreißen, Fasten, Einnehmen von Abführmitteln usw. " die Anwendung des Kabinettsbeschlusses nicht rechtfertigen könnten. Vgl. die Schriftwechsel der Rechtsanwaltskanzlei Bastian, Müller-Meiningen, Nowak, Kückelmann mit dem Bundesfinanzministerium vom 7.9. 1960, 5.2. 1963, 12.3. 1963, 27. 1. 1965, PA/AA, B 126/61085. Die Antragsteller entstammten der jüdischen Familie Ovici, die als Liliputaner-Gruppe in ganz Europa gastierte. Im Mai 1944 wurde die ganze Familie nach Auschwitz. deportiert. Vgl. auch die Aufzeichnungen über das Schicksal der Familie von Moshkovitz in dies., By Grace of the Satan.

18 „Abscheu vor NS-Versuchen. Familienministerin Süssmuth im Gespräch mit dem Vorsitzenden der jüdischen Gemeinde Galinski“, die tageszeitung vom 12.11.1986, S.2.

19 „Mengele-Opfer“, die tageszeitung vom 25.6.1987, S.5.

20 Der Präsident der Max-Planck-Gesellschaft hatte im Juni 2001 ein Symposium zum Thema „Biowissenschaften und Menschenversuche am Kaiser-Wilhelm-Institut" veranstaltet und dazu Überlebende der Zwillingsforschung eingeladen. Vgl. dazu: Hubert Leber, „Wir haben uns der Aufklärung lange entzogen. Die Max-Planck-Gesellschaft untersucht ihre Verstrickungen in die Menschenversuche in Auschwitz.“, Berliner Zeitung vom 11.6.2001; Hans Schuh, „Biomedizin in Auschwitz. Der Max-Planck- 
Der Stein war durch eine ungewöhnliche Initiative ins Rollen gekommen. Schon seit einigen Jahren ging der Fall von Eva Mozes Kor, die mit ihrer Zwillingsschwester die Experimente in Auschwitz überlebt hatte und sich für eine Anerkennung der Opfer einsetzte, durch die Presse. Besonders spektakulär war eine von ihr angestrengte Sammelklage gegen den Chemie- und Pharmakonzern Bayer im Jahr 1999. ${ }^{21}$ Kor, die mit ihrer Initiative Children of Auschwitz-Nazi's Deadly Lab Experiments Survivors 122 Überlebende von Zwillingsexperimenten ausfindig gemacht und sogar ein Museum für die Opfer der medizinischen Versuche in der Nazi-Zeit gegründet hatte, ${ }^{22}$ ging in ihrem Engagement für die Leidensgenossen jedoch noch einen wesentlichen Schritt weiter.

In öffentlichen Auftritten plädierte sie dafür - nachdem die Wahrheit ans Licht gebracht sei -, das „Streben nach Gerechtigkeit und Rache“ aufzugeben, denn „geheilt worden vom Schrecken der Vergangenheit sei sie erst, nachdem sie den Tätern von damals habe verzeihen können “. ${ }^{23}$ Als Überlebende von Mengeles grausamen Humanexperimenten trat sie damit für Vergebung und Versöhnung mit den Tätern ein, da es das Recht der Betroffenen sei, die Opfer-Rolle aufzukündigen und damit die eigene Autonomie zurückzugewinnen. ${ }^{24}$

Eva Mozes Kor löste vor allem bei anderen Holocaust-Überlebenden kritische Reaktionen aus. So kommt in dem amerikanischen Dokumentarfilm „Forgiving Dr. Mengele“, der die Bewältigungsstrategie von einer „verbitterten Überlebenden“ zu einer „unermüdlichen Anwältin der Versöhnung" aufzeigt, ein anderes Opfer der Zwillingsexperimente zu Wort. Die Überlebende wirft Kor darin vor, dass sie etwas verzeihe, das nicht nur ihr, sondern auch vielen anderen angetan worden sei. ${ }^{25}$ Über die Möglichkeiten und die grundlegende Bedeutung von Vergebung entzündete sich im Zusammenhang mit den umstrittenen Bewältigungsstrategien von Eva Mozes Kor aber auch eine öffentliche Diskussion, vor allem in der Sozialpsychologie sowie in der Trauma- und Biographieforschung. ${ }^{26}$

Präsident entschuldigt sich bei den Opfern“, Die Zeit vom 13.6. 2001, S.31; Thomas Fitzel, „Die Experten klären auf. Die Max-Planck-Gesellschaft entschuldigt sich“, die tageszeitung vom 11.6. 2001, S. 14.

${ }^{21}$ „Mengele-Opfer verklagt Bayer wegen Experimenten“, Süddeutsche Zeitung vom 19.2. 1999; Stefan Kornelius, „Was die Hölle kostet. Sie überlebte Mengeles Zwillingsexperimente, nun klagt die Amerikanerin Eva Kor gegen Bayer“, Süddeutsche Zeilung vom 7.4.1999, S. 3 u. S. 7; „Die Erinnerung der Täter“, Der Spiegel vom 28. 9. 1998; Hans Halter, „Erinnerung an den Teufel“, Der Spiegel vom 11.6.2001.

${ }^{22}$ Das Museum befindet sich in Terre Haute, Indiana. Informationen über das Museum findet man auf der Homepage unter http://www.candlesholocaustmuseum.org (5.1.2009).

${ }_{23}$ Vgl. Interview Hubert Lebers mit Eva Mozes Kor, „Wir haben uns der Aufklärung zu lange entzogen. Die Max-Planck-Gesellschaft untersucht ihre Verstrickungen in die Menschenversuche in Auschwitz.", Berliner Zeitung vom 11.6.2001; Kai Michel, „Gleich an Ort und Stelle in Formol fixiert“, Berliner Zeitung vom 5.12.2001, S. 18 .

${ }^{24}$ 'Vgl. „Wahrheit heilt. Wie eine Auschwitz-Überlebende die Zwillingsversuche der Nazis bewältigt.“ Gekürzte Fassung der Rede, die Eva Mozes Kor anlässlich der Eröffnung des Symposiums „Biowissenschaften und Menschenversuche an Kaiser-Wilhelm-Instituten" hielt, Die Zeit vom 13.6. 2001, S.34f.; siehe auch Kor, Heilung von Auschwitz, S. 68.

25 Der Dokumentarfilm „Forgiving Dr. Mengele" von Bob Hercules und Cheri Pugh wurde im Jahr 2005 produziert und erstmalig am 6.12.2005 in Deutschland im KörberForum/Hamburg gezeigt. Vgl. dazu Georg Felix Harsch, „Zweifelhafte Vergebung. ,Forgiving Dr. Mengele‘: Der Dokumentarfilm Cheri Pughs jetzt im KörberForum zu sehen", die tageszeitung vom 1. 12.2005, S. 23.

${ }^{26} \mathrm{Zu}$ den Diskussionen zum Thema „Vergebung“ vgl. das Interview Harald Welzers mit Eva Mozes Kor, „Ein Überlebender hat das Recht zu vergeben“, Frankfurter Rundschau vom 13.6.2003, S.2; Micha Brumlik/Lena Inowlocki, „Die grundlegende Bedeutung der Vergebung. Antwort auf Harald Welzers Umgang mit der Trauma-Therapie“, Frankfurter Rundschau vom 23.6. 2003, S. 8; Harald Welzer, "Vergeben ist ein Recht aller Opfer", Frankfurter Rundschau vom 30.6.2003, S.8. 
Doch selbst wenn man aus einem theologischen Verständnis von „Schuld“ und „Versöhnung" davon ausgeht, dass Vergebung der Teil ist, den nur die Opfer oder deren Angehörige als Voraussetzung für eine Versöhnung leisten können, so setzt das voraus, dass die Schuldigen tun, was in ihrer Macht steht, um die Folgen der verbrecherischen Taten zu tilgen. ${ }^{27}$ Das Eingestehen der Schuld, das dafür notwendig ist, bedingt wiederum das gemeinsame Erinnern der Tat als „Geheimnis der Erlösung“ ${ }^{28}$ Und genau daran mangelte es über Jahrzehnte auf der Seite der Schuldigen.

Die Opfer leisteten - wenn auch spät - ihren Beitrag zur Erinnerung und zur Wiedergutmachung. Denn es waren gerade die öffentlichen Auftritte ehemaliger Verfolgter mit ihren emotionalen Stellungnahmen, die dazu führten, dass in einigen Fällen die diplomatische Verhandlungsroutine durchbrochen wurde. So hatte das Ravensbrueck Lapins Project bereits Anfang der 1960er Jahre gezeigt, dass die mediale Inszenierung der polnischen Frauen in der amerikanischen Öffentlichkeit große Wirkung zeigte und damit auch für die Wiedergutmachung eine erhebliche Schubkraft entwickelte. Während der Opferstatus in dieser Zeit noch eher verpönt war, ist er heute für Gruppen unterschiedlichster Art eine moralische und politische Basis geworden, von der aus sich wirksam agieren lässt. ${ }^{29}$ Seit den 1990er Jahren sind „solche auf den Eindruck der Authentizität setzenden Auftritte von survivors [...] selbst zum Teil medialer Inszenierung insbesondere seitens der class-actionAnwälte". 30

Die Voraussetzung dafür schufen die weltpolitischen Veränderungen von 1989/90. Das Ende des Ost-West-Konflikts und die Vereinigung Deutschlands bezeichnen eine epochale Zäsur, die auch in der Wiedergutmachungsgeschichte zu einer neuen Bewegungs- und Gestaltungsphase führte - und zwar vor allem Richtung Ost. Nach dem Muster der Zwei-plusVier-Abkommen von September 1990 wurden mit Polen und mit drei Nachfolgestaaten der Sowjetunion (Russische Föderation, Ukraine und Weißrussland) Globalabkommen abgeschlossen. Ebenso wurden Vereinbarungen mit den drei baltischen Staaten getroffen sowie der Deutsch-Tschechische Zukunftsfonds gegründet. ${ }^{31}$ Diese Wiederbelebungswelle von Entschädigungsfragen ist wiederum nur verständlich, „wenn man sich vor Augen hält, wie begrenzt die Wiedergutmachung im Zeitalter des Ost-West-Konflikts und der deutschen Teilung gewesen war".32

Aber parallel dazu baute sich - ausgelöst von Sammelklagen gegen Schweizer Banken vor US-amerikanischen Gerichten - eine weitere Welle auf, die in den Jahren 1997 und 1998 Deutschland crreichte. ${ }^{33}$ Zunächst waren Banken und Versicherungen betroffen, ${ }^{34}$ dann deutsche Industrieunternehmen, die in der NS-Zeit von ausländischen Zwangsarbeitern profitiert hatten. Zwar waren entsprechende Entschädigungsforderungen, die bereits seit

27 Dieser Aspekt wird auf rechtsphilosophischer Ebene ausführlich diskutiert in: Schlink, Vergangenheitsschuld, S. $101 \mathrm{f}$.

${ }^{28}$ Schwencke, Geheimnis der Versöhnung.

29 Zur Umwertung des Opferstatus vgl. Levy/Sznaider, Erinnerung, S. 107f.

${ }^{30}$ Goschler, Die Bundesrepublik und die Entschädigung von Ausländern, S. 135.

$31 \mathrm{Zu}$ den verschiedenen Abkommen mit osteuropäischen Staaten vgl. Hockerts, Entschädigung, S. 50-52; Goschler, Die Bundesrepublik und die Entschädigung von Ausländern, S. 117-124.

32 Hockerts, Entschädigung, S. 56.

33 Zwei große internationale Konferenzen lenkten die Aufmerksamkeit in diesen Jahren auf die Verstrickungen verschiedener Länder in die NS-Raubpolitik, zu diesem Thema siehe auch die verschiedenen Aufsätze in Goschler/Ther (Hrsg.), Raub und Restitution.

${ }^{34}$ So standen verschiedene Versicherungen in dem Verdacht, Policen von Holocaust-Opfern nicht ausgezahlt zu haben. Vgl. Hockerts, Entschädigung, S.54. 
den 1950er Jahren erhoben wurden, mit wenigen Ausnahmen immer wieder erfolgreich abgeschmettert worden, jetzt aber hatten sich die Koordinaten im Zuge der Globalisierung entscheidend zu Ungunsten der Bundesrepublik verändert. Die deutsche Wirtschaft sah sich den aggressiven und medienwirksamen Agitationen der class-action-Anwälte in den USA ausgesetzt, die nicht nur juristisch gefährlich werden konnten, sondern vor allem die öffentliche Meinung international negativ zu beeinflussen imstande waren.

So wurden die Entschädigungsverhandlungen nicht mehr - wie in den Jahrzehnten zuvor - individuell oder bilateral, sondern vor dem Tribunal der Weltöffentlichkeit ausgetragen. Zwar ist das Instrument der Sammelklage als juristische Plattform, um politisch brisante Themen aufzudecken, nicht unumstritten, dennoch war es für die „Benennung von blinden historischen Flecken " zunächst unersetzlich - so jedenfalls sieht es Stuart Eizenstat, der Chefunterhändler der US-Regierung bei den Sammelklagen von ehemaligen Zwangsarbeitern und anderen NS-Opfern gegen Schweizer Banken, Versicherungen und die deutsche Industrie retrospektiv. ${ }^{35}$ Dem Schreckensszenario von Boykottaufrufen, weltweitem Imageverlust und den daraus resultierenden wirtschaftlichen Folgeschäden begegnete die deutsche Politik gemeinsam mit der Industrie im Jahr 1998 mit der Stiftungsinitiative der deutschen Wirtschaft ${ }^{36}$ - ein Novum in der bisher ganz vom Staat und mit staatlichen Geldern getragenen Entschädigungspolitik. Zwar spielte auch hier einmal mehr der amerikanische Druck eine entscheidende Rolle, aber bei der neuen rot-grünen Regierung war durchaus auch eine grundsätzliche Bereitschaft spürbar, offene Wiedergutmachungsfragen zu regeln. ${ }^{37}$

In zahlreichen schwierigen Verhandlungsrunden versuchten die deutschen Verhandlungspartner den Spagat, Verantwortung für geschehenes Unrecht zu übernehmen und gleichzeitig den Rechtsfrieden für die deutschen Unternehmen zu sichern. Am 6.Juli 2000 beschloss der Deutsche Bundestag dann mit breiter Mehrheit die Errichtung der Stiftung „Erinnerung, Verantwortung und Zukunft“. Wenige Tage später trat das Stiftungsgesetz, demzufolge der deutsche Staat und die deutsche Wirtschaft jeweils fünf Milliarden DM in den Fonds einbringen sollten, mit der Unterzeichnung einer internationalen Abschlusserklärung in Kraft. Ende des 20. Jahrhunderts hatten sich damit sowohl die Akteure als auch die Aktionsformen in der Wiedergutmachung entscheidend gewandelt: von den „klassischen" bilateralen Globalabkommen der 1950er und 1960er Jahre hin zu einer globalisierten Entschädigungspolitik. ${ }^{38}$

Der Hauptzweck des Fonds lag darin, die etwa 1,7 Millionen noch lebenden ehemaligen Zwangsarbeiter zu entschädigen. Aber auch für andere von NS-Unrecht betroffene Personen sollten Leistungen bereitgestellt werden. Entsprechend wurde der weitaus größte Anteil mit 8,1 Milliarden DM für die Entschädigung von Zwangsarbeitern aufgebracht. Darüber hinaus waren 1,1 Milliarden DM für Vermögensschäden sowie 50 Millionen DM für Verfolgungsopfer, die unter die Kategorie „sonstige Personenschäden“ fielen, vorgesehen - und zwar vorrangig für die Opfer von Humanexperimenten, bei denen es ganz offensichtlich immer noch „Nachsteuerungsbedarf" gab. ${ }^{39}$

35 Eizenstat, Unvollkommene Gerechtigkeit, S. 102-119.

36 Spiliotis, Verantwortung und Rechtsfrieden; Saathoff, Entschädigung für Zwangsarbeiter?.

$37 \mathrm{Zu}$ den Initiativen der neuen Regierung unter Gerhard Schröder vgl. Goschler, Schuld und Schulden, S. 134.

38 Goschler, Die Bundesrepublik und die Entschädigung von Ausländern, S. 143.

39 Unter die Kategorie fielen auch die sogenannten Zwangsarbeiterkinderfälle. 
Die im Jahr 1954 abgegebene Prognose des Bundesfinanzministeriums, dass die Wiedergutmachung zugunsten dieser Opfergruppe in kürzester Zeit abschließend geregelt sei, erwies sich erneut als Fehleinschätzung. ${ }^{40}$ Im Laufe der Jahrzehnte war es immer wieder zu Kritik gekommen, immer wieder musste nachgebessert werden, und immer wieder tauchten neue Fälle auf, die Rückschlüsse auf bis dahin unbekannte oder nicht anerkannte Verbrechenstatbestände gaben. So öffnete sich fast 50 Jahre später - im Jahr 2000 - noch einmal eine „Falltür in die Vergangenheit“. ${ }^{41}$

Mit der Errichtung der Stiftung „Erinnerung, Verantwortung und Zukunft“ erachtete man es ganz offensichtlich für notwendig, die bisherige Wiedergutmachungspraxis auch für diese Opfergruppe noch einmal zu ergänzen, um den Überlebenden in aller Welt schnelle finanzielle Hilfe zu gewähren. Aus Gründen der Verfahrenserleichterung wurden im Vergleich zur bisherigen Praxis keine sogenannten harten Beweismittel verlangt, sondern es genügte das Prinzip der „Glaubhaftmachung“. Nach nunmehr beinahe 60 Jahren sollte es nicht mehr darum gehen, die Wahrheit vollständig aufzuklären, sondern mit Blick auf das Alter der überlebenden Opfer die - vermutlich letzte - Chance zu ergreifen, wohlwollend zu agieren, so der Stiftungsvorstand Günter Saathoff. ${ }^{42}$ Als Grundlage für die Anerkennung eines Personenschadens wurde eine ausführliche Liste erstellt, in der nicht nur Ort und Art der Menschenversuche angegeben werden, sondern auch die Täter und der Zeitraum, in dem die Humanexperimente durchgeführt worden waren. Ferner sind in der sechsseitigen Übersicht, die 40 verschiedene Orte und insgeamt 197 medizinische Versuchsanordnungen nennt, auch Quellen und Dokumente verzeichnet. ${ }^{43}$

Aufgrund der zahlreichen Anträge wurde der Fonds aus Zinsmitteln der Bundesstiftung sogar noch einmal aufgestockt. ${ }^{44}$ Laut den Statistiken der Stiftungsinitiative erhielten bis Ende des Jahres 2004 rund 6700 überlebende Opfer von Menschenversuchen mit Hilfe der Partnerorganisationen in den verschiedenen Ländern sowie über die Vermittlungsarbeit internationaler Organisationen einen einmaligen Pauschalbetrag von gut 4200 Euro. ${ }^{45}$

40 Im Bundesfinanzministerium ging man im März 1954 davon aus, dass „die Aktion im Auslaufen begriffen" sei und die genehmigten 500000 DM für 1954 ausreichen würden. Für den Fall, dass noch einige Anträge nachgereicht werden sollten, könnten noch $100000 \mathrm{DM}$ für das Jahr 1955 bereitgestellt werden. Vgl. Aufzeichnung einer internen Besprechung im BMF vom 23.3.1954, BA, B 126/12553.

41 Mit dieser Formulierung spielte Hans Günter Hockerts, der die Sektion „Grenzen und Räume der Wiedergutmachung“ auf dem Kieler Historikertag im Jahr 2004 leitete, auf Gegenwartsbezüge im Rahmen der Wiedergutmachungsgeschichte an, „deren historische Klärung und Einordnung zu den großen Aufgaben der Zeitgeschichte zählt." Konkret bezog er sich hier auf Äußerungen des polnischen Parlaments vom September 2004, Polen habe bislang keine angemessene finanzielle Kompensation und Kriegsreparationen für die durch die deutsche Aggression verursachten Zerstörungen sowie die materiellen und immateriellen Verluste erhalten. Vgl. den Tagungsbericht von Tobias Winstel vom Historikertag 2004: Grenzen und Räume der Wiedergutmachung. Die Entschädigung für NS Verfolgte in West- und Osteuropa, http://hsozkult.geschichte.hu-berlin.de/tagungsberichte/id=436 (5. 1.2009).

42 Gespräch der Autorin mit Günter Saathoff vom 15. 12.2004.

43 Aufgelistet sind neben den bekannten Konzentrationslagern auch die Nebenlager sowie verschiedene Institute und mehrere Lager in Transnistrien. Die Übersicht ist zu finden unter: http://www. stiftung-evz.de/content/view/5/18/.

${ }^{44}$ Vierter Bericht der Bundesregierung über den Stand der Auszahlungen und die Zusammenarbeit der Stiftung „Erinnerung, Verantwortung und Zukunft“ mit den Partnerorganisationen, BT-Drucksache, $15 / 3440,26.6 .2004$.

45 Anfangs waren 7000 DM pro berechtigter Person eingeplant, nach der Aufstockung ergab sich ein Betrag von 8300 DM (4243,72 Euro); laut Gespräch mit Stiftungsvorstand Günter Saathoff vom 15. 12. 2004 sah die Verteilung der Fälle mit Stand Dezember 2004 wie folgt aus: 272 Belarus (Estland), 
Es wurde erreicht, was man sich vorgenommen hatte: Die finanziellen Mittel kamen innerhalb vergleichsweise kurzer Zeit zur Auszahlung. Und man hatte mit dem Stiftungsgesetz das strategische Ziel der deutschen Wirtschaft erreicht, sich Rechtssicherheit zu erkaufen, da ein „Quasi-Schlussgesetz“ für eine Vielzahl von unbefriedigten Ansprüchen geschaffen wurde. ${ }^{46}$

Susanne-Sophia Spiliotis geht in ihrer aus der Innenansicht verfassten Darstellung der Geschichte der Stiftungsinitiative so weit, auf einen letztendlich gelungenen Interessenausgleich zu schließen, bei dem Zwangsarbeiter und deutsche Wirtschaft endlich ihren Rechtsfrieden gefunden hätten. ${ }^{47}$ Anders sieht das Lutz Niethammer, der von 1998 bis 2000 als historischer Berater der Bundesregierung bei der Vorbereitung der Entschädigung für Zwangsarbeiter und den dazu geführten internationalen Verhandlungen mitwirkte. Zwar bewertet er die Stiftungsinitiative als einen kulturellen Fortschritt im Sinne der Erinnerung von Anerkennung und Verantwortung, einen wesentlichen Rechtsfortschritt vermag er darin allerdings nicht zu erkennen. ${ }^{48}$

Auch in diesem Punkt wiederholt sich einmal mehr die Geschichte der Wiedergutmachung: Während die einen selbstbewusst darauf verweisen, dass die Schuld wenigstens materiell beglichen und mit der Solidaraktion der lang ersehnte Schlussstrich gezogen sei, präsentieren Kritiker offene Rechnungen und kommentieren die Verhandlungstaktik der deutschen Konzerne zynisch als „Endlösung der Entschädigungsfrage“ oder „final insult“. Moderater und viel treffender spricht Stuart Eizenstat in seiner 2003 erschienenen Studie von der „unvollkommenen Gerechtigkeit“. ${ }^{9}$

Die Bezieher der Leistungen aus der Stiftungsinitiative mussten eine Erklärung unterschreiben, wonach alle weiter gehenden Ansprüche ausgeschlossen wurden. ${ }^{50}$ Nachdem bis Mitte des Jahres 2005 über 98 Prozent der Fälle abgewickelt waren, scheint damit auch das Kapitel „Wiedergutmachung für Opfer von Menschenversuchen“ abgeschlossen zu sein.

770 IOM, 2527 JCC (Jewish Claims Conference), 1207 Polen, 96 Russland (mit Lettland und Litauen), 157 Tschechien, 1313 Ukraine.

46 Saathoff, Entschädigung für Zwangsarbeiter?, S. 248.

47 Spiliotis, Verantwortung und Rechtsfrieden, S. 205.

48 Niethammer, Beschädigte Gerechtigkeit.

49 So zum Beispiel Tjark Kunstreich, „Endlösung der Entschädigungsfrage. Der Fonds der Bundesregierung wird deutsche Konzerne, die vom KZ-System profitierten, endgültig von allen Forderungen befreien“, Jungle World vom 10.3.1999; siehe auch die gruppe offene rechnungen (Hrsg.), Final Insult; Eizenstat, Unvollkommene Gerechtigkeit.

${ }^{50} \S 16,2$, Gesetz. zur Errichtung der Stiftung „Erinnerung, Verantwortung und Zukunft“. 